\title{
A Predefined Bit-Plane Comparison Coding for Mobile Video Applications
}

\author{
Chien-Chen Lin, Yao Li, and Chen-Yi Lee, Member, IEEE
}

\begin{abstract}
For mobile video applications, the required storage and bandwidth of frame memory play crucial roles. Reducing the accesses and the size of frame memory can decrease area and cost, as well as power consumption. Current video-coding standards can reach high compression efficiency but demand large volumes of computations and storage requirements. Hence, it is hard for these coding standards to be embedded into an $\mathbf{H . 2 6 4}$ decoder. In this brief, a novel embedded lossy compression scheme is proposed. With a compression ratio at 2 , it compresses a $4 \times$ 2 size block into 32 bit with peak-signal-to-noise-ratio loss of $1.27 \sim 3.94 \mathrm{~dB}$ compared with H.264. A pipelined architecture is also proposed to realize both the compressor and the decompressor in 2 cycles and 1 cycle, respectively. This cost-effective solution requires a gate count of $4.9 \mathrm{k}$ and power consumption of $244 \mu \mathrm{W}$ in a 90-nm standard complementary metal-oxide-semiconductor process, making it very suitable for mobile video applications.
\end{abstract}

Index Terms-Embedded compression (EC), H.264, lossy compression, memory reduction, pattern comparison.

\section{INTRODUCTION}

$\mathbf{V}$ IDEO-CODING standards achieve high compression efficiency such as H.264 [1] and so forth. For H.264, at least one previous frame is stored in the external memory device to generate a predicted frame. Obviously, motion compensation (MC) demands a huge amount of data accesses between offchip memory devices and the video decoder chip. However, data transfer consumes a lot of power. For mobile video devices, one major issue is the limited power supply from the battery. It is essential to reduce the bandwidth requirement and the size of frame memory while maintaining acceptable visual quality. Therefore, embedded compression (EC) is a suitable technique to achieve the requirement.

EC methods can be categorized majorly into two types: lossless and lossy. Lossless compression algorithms [2], [3] have the advantages of no error propagation and quality loss. However, the variable lengths of the compressed data result in irreducible frame-memory size. Hence, existing lossless algorithms are not suitable for frame compression because their primary purpose is to achieve high coding efficiency rather than low latency, computation complexity, and high random accessibility.

Manuscript received October 6, 2010; revised January 9, 2011 and March 8, 2011; accepted April 25, 2011. Date of publication June 27, 2011; date of current version July 20, 2011. This work was recommended by Associate Editor B.-D. Liu.

The authors are with the Department of Electronics Engineering \& Institute of Electronics, National Chiao Tung University, Hsinchu, Taiwan (e-mail: matriclin@si2lab.org; yaoli@si2lab.org; cylee@si2lab.org).

Digital Object Identifier 10.1109/TCSII.2011.2158271
Lossy compression algorithms, compared with lossless compression algorithms, accomplish the fixed compression ratio (CR). Several lossy compression algorithms have been proposed, such as the modified Hadamard transform (MHT) with the quantization of Colomb-Rice coding [4], discrete cosine transform with modified bit-plane zonal coding [5], and so on. Yang and Chai [6] exploit 64 patterns to improve block truncation coding, where Amarunnishad et al. [7] try to enlarge acceptable quality loss by reducing the number of compared patterns in [6]. Lossy compression algorithm with the fixed CR can guarantee the reduction of frame-memory size. However, how to maintain an acceptable visual quality remains to be solved. Consequently, it is important to design a lossy algorithm with the following features: 1) low-distortion visual quality; 2) low complexity; 3) low bandwidth requirement; and 4) low power consumption.

In this brief, a novel embedded lossy algorithm based on predefined bit-plane comparison coding (PBCC) is proposed. The CR is fixed at 2 . Each $4 \times 2$ block can be compressed into a 32-bit segment. The proposed PBCC encodes a $4 \times 2$ block in 2 cycles and decodes a $4 \times 2$ block in 1 cycle to meet the requirement of embedded frame data processing.

The rest of this brief is organized as follows. In Section II, a novel algorithm is described briefly. The hardware architecture, which is suitable for mobile video applications, is given in Section III. Sections IV and V discuss the integration with an available H.264 decoder [8] and the experimental results, respectively.

\section{Proposed EC Algorithm}

The proposed algorithm compresses a $4 \times 2$ block ( 64 bit) from the output of deblocking filters. The CR is fixed at 2, implying that a $4 \times 2$ block will become a 32-bit segment after compression. With fixed CR, the amount of the coded data is constant. Therefore, this compression can guarantee access times. In addition, in the H.264 standard, a $4 \times 4$ block, which is a basic coding unit, can be partitioned into two $4 \times 2$ blocks.

Fig. 1 shows the flowchart of the proposed compression algorithm. We divide the algorithm into four parts: 1) pixel truncation; 2) selective bit planes; 3 ) rounding; and 4) a pattern comparison. These parts will be described in the following. The compressed 32-bit-segment format is shown in Fig. 2. The representation format consists of a 2-bit mode, a 2-bit start plane (SP), a 2-bit pattern L, a 2-bit pattern R, 12-bit coded data L, and 12-bit coded data R. The mode indicates the selected mode out of four modes, and the SP is derived 


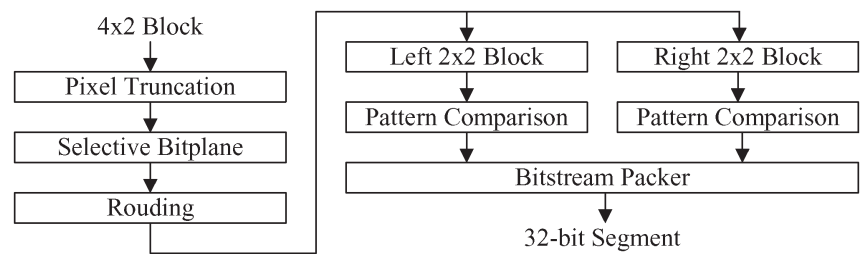

Fig. 1. Compression flow of the proposed algorithm.

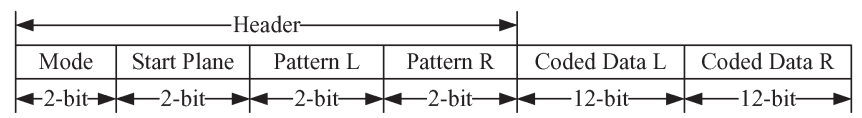

Fig. 2. Compressed 32-bit-segment format.

TABLE I

Three Groups OF EIght PATTERnS

\begin{tabular}{|c|c|c|c|c|c|c|c|c|}
\hline Pattern No. & 1 & 2 & 3 & 4 & 5 & 6 & 7 & 8 \\
\hline Group A & 0000 & 1111 & 1110 & 0111 & 0011 & 1100 & 0001 & 1000 \\
\hline Group B & 0000 & 1111 & 1110 & 0111 & 1010 & 1001 & 0110 & 0101 \\
\hline Group C & 0000 & 1111 & 1110 & 0111 & 1101 & 1011 & 0010 & 0100 \\
\hline
\end{tabular}

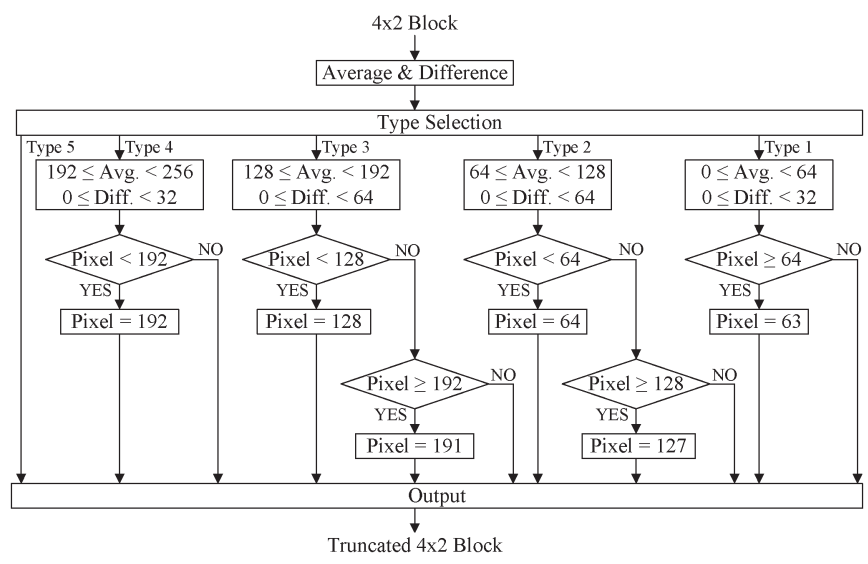

Fig. 3. Flowchart of the pixel truncation.

from the bit-plane selection of the selected mode, as shown in Fig. 4. Patterns $\mathrm{L}$ and $\mathrm{R}$ indicate which group is selected to be compared with the left and right $2 \times 2$ blocks, as shown in Fig. 6. In Table I, coded data $L$ and $R$ pack four patterns or three successive bit planes for the left $2 \times 2$ block and the right $2 \times$ 2 block, respectively.

\section{A. Pixel Truncation}

Fig. 3 shows the flowchart of the pixel truncation. First, we calculate the average value (Avg.) of the $4 \times 2$ block and the difference value (Diff.) between the maximum pixel and the minimum pixel of the $4 \times 2$ block. Second, according to Avg. and Diff., we classify those $4 \times 2$ subblocks into five types as follows.

1) Avg. is from 0 to 63, and Diff. is smaller than 32;

2) Avg. is from 64 to 127, and Diff. is smaller than 64;

3) Avg. is from 128 to 191, and Diff. is smaller than 64;

4) Avg. is from 192 to 255, and Diff. is smaller than 32;

5) No change.
In type 1 , if each pixel is larger than or equal to 64 , we force the pixel to be 63 . In type 2 , if each pixel is less than 64 , we force the pixel to be 64; if each pixel is larger than or equal to 128 , we force the pixel to be 127. Types 3 and 4 are processed such as types 2 and 1, respectively. In type 5, the original pixel value remains unchanged.

\section{B. Selective Bit Plane}

Fig. 4 shows the flowchart of the selective bit plane. Bit-plane coding is a well-known method. We exploit the bit plane as a basic unit to a group of numbers, instead of a pixel-wised basic unit. First, we consider a $4 \times 2$ block in which each pixel value is represented by 8 bit. A bit plane can be formed by selecting a single bit from the same position in the binary representation of each pixel. We define that $\mathrm{B} 7$ represents the most significant bit (MSB) plane, whereas B0 represents the least significant bit (LSB) plane. Second, the SP is searched for four successive bit planes from the MSB plane with four modes as follows: 1) B7, $\mathrm{B} 6$, and $\mathrm{B} 5$ are $0 \times 00 ; 2) \mathrm{B} 6$ is $0 \times \mathrm{FF}$, and $\mathrm{B} 7$ and $\mathrm{B} 5$ are $0 \times$ 00 ; 3 ) $\mathrm{B} 7$ is $0 \times \mathrm{FF}$, and $\mathrm{B} 6$ and $\mathrm{B} 5$ are $0 \times 00$; and 4) $\mathrm{B} 7$ and $\mathrm{B} 6$ are $0 \times \mathrm{FF}$, and $\mathrm{B} 5$ is $0 \times 00$.

In mode 1, if both B7 and B6 are larger than $0 \times 00$ and B5 is equal to $0 \times 00$, the $\mathrm{SP}$ is equal to 2 . Similarly, the other modes act like mode 1. Finally, the maximum SP is selected from four modes.

\section{Rounding}

Since lower bit planes are truncated due to the limited budget, a simple rounding is applied here. When the significant bit of the truncated bits is nonzero and the coded bits are not all in the value of 1, the rounding is applied to each pixel. In Fig. 5(a), this rounding technique can be considered as adding a median number of a lost bit plane. This idea leads to a satisfied quality improvement. Two rounding modes are proposed because the pattern comparison has two data compressed formats. As shown in Fig. 5(b), the first rounding is the comparison code rounding, and the second rounding is the no comparison rounding. For the pattern comparison, the first rounding method is applied to the first three cases, and the second rounding method is only for the last case.

\section{Pattern Comparison}

The last step encodes the preserving bit planes. First, the truncated $4 \times 2$ block is partitioned into two $2 \times 2$ blocks, which are called the left $2 \times 2$ block and the right $2 \times 2$ block, as shown in Fig. 6(a). In Fig. 6(b), both the left $2 \times 2$ block and the right $2 \times 2$ block exploit the same SP for compression. Second, four cases for a $2 \times 2$ block are classified as: 1) group A; 2) group $B$; 3) group $C$; and 4) no comparison. Through simulation and statistics analysis, the $2 \times 2$ patterns with a higher hit rate are collected to form one group. The first three cases exploit a group of eight patterns to compare with four successive bit planes from the SP and select one case that can hit three successive bit planes. The three groups of the eight patterns are shown in Table I. If the first three cases are not 


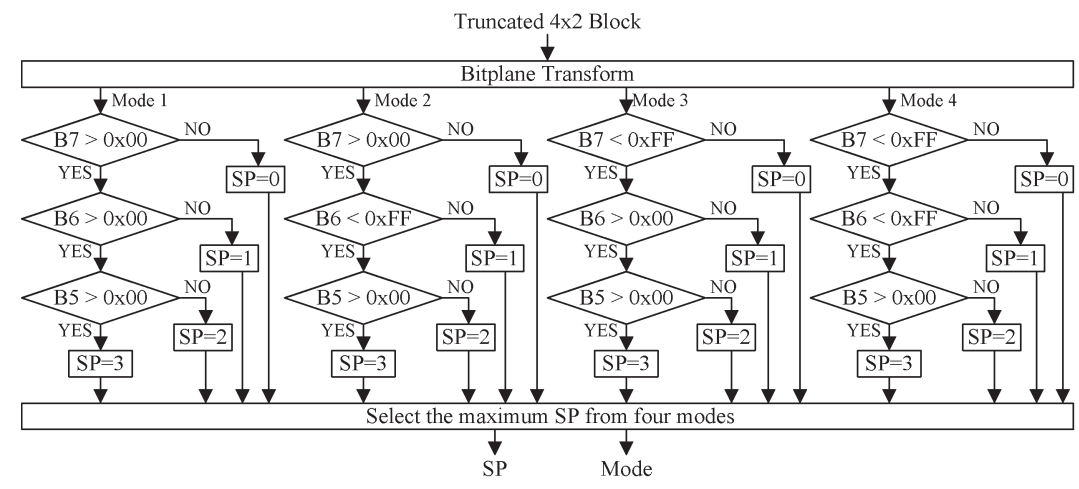

Fig. 4. Flowchart of the selective bit plane.

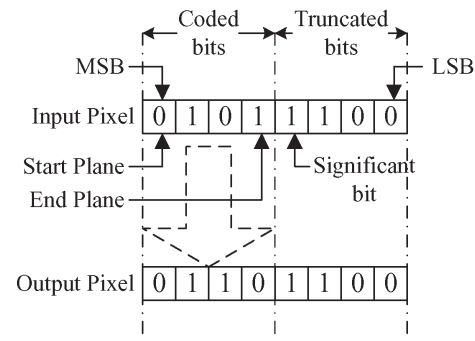

(a)

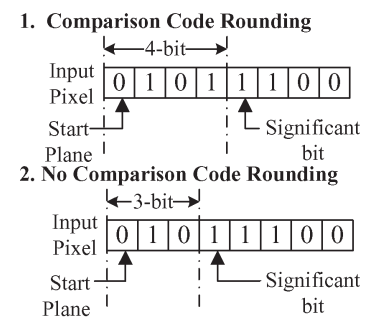

(b)

Fig. 5. Flowchart of the rounding.

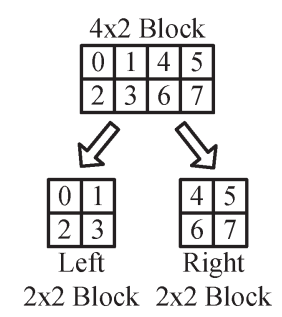

(a)

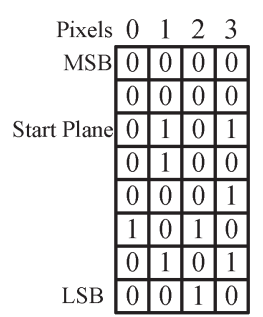

(b)

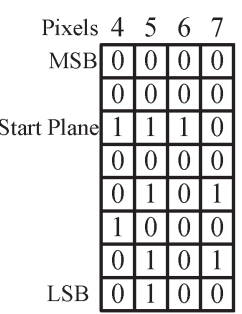

Fig. 6. Example of partitioning the $4 \times 2$ block.

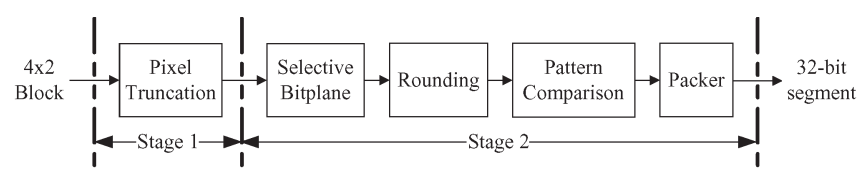

Fig. 7. Compressor architecture.

matched with these three bit planes, case 4, i.e., no comparison, is chosen, and three successive bit planes from the SP are then stored.

\section{Proposed Architecture}

\section{A. Compressor Design}

Fig. 7 shows the pipeline architecture of this compressor design. We use two pipeline stages, and each of which requires one cycle. The first stage is the pixel truncation. The second stage is composed of selective bit planes, rounding, a pattern comparison, and a packer. This compressor encodes a $4 \times 2$ block in 2 cycles.

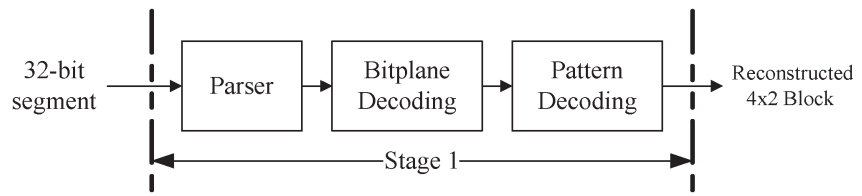

Fig. 8. Decompressor architecture.

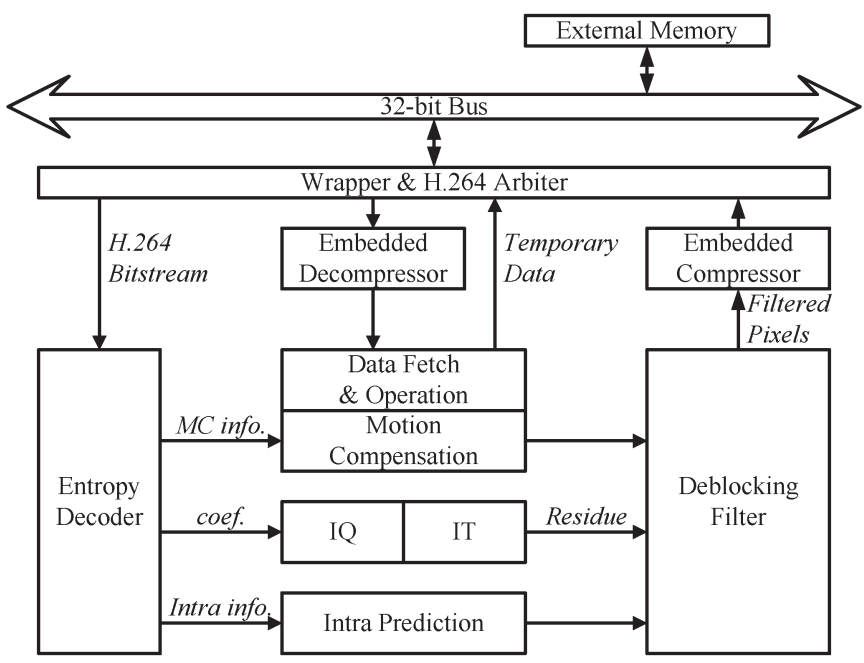

Fig. 9. H.264 decoder with the proposed EC.

\section{B. Decompressor Design}

Fig. 8 shows the pipeline architecture of the decompressor. The decompressor only needs one stage with one cycle for parsing, bit-plane decoding, and pattern decoding. This decompressor can achieve high throughput, leading to better random accessibility compared with other designs.

\section{System InTEGRATION}

The overall H.264 decoder with the EC codec is shown in Fig. 9. The data path of the embedded compressor and the decompressor is from the deblocking filter to the external memory device and from the external memory device to MC, respectively. The address controller of EC is simple since the $\mathrm{CR}$ is fixed at 2. Our system bus is 32 bit, and the external memory device is 32 bit per entry. 
TABLE II

All Cases of Read Access Requirement

\begin{tabular}{|c|c|c|c|}
\hline Case of MV (x, y) & $\begin{array}{c}\text { Access } \\
\text { Cycles for } \\
\text { System } \\
\text { without EC }\end{array}$ & $\begin{array}{c}\text { Access Cycles } \\
\text { for System } \\
\text { with } \\
\text { Proposed EC }\end{array}$ & $\begin{array}{c}\text { Reduction of } \\
\text { Access } \\
\text { Cycles } \\
\text { without EC }\end{array}$ \\
\hline (Align, Align) & 4 & 2 & 50 \\
\hline (Align, Not Align) & 4 & $2 / 3$ & $50 / 25$ \\
\hline (Align, Sub) & 9 & 5 & 44.4 \\
\hline (Not Align, Align) & 8 & 4 & 50 \\
\hline (Not Align, Not Align) & 8 & $4 / 6$ & $50 / 25$ \\
\hline (Not Align, Sub) & 18 & 10 & 44.4 \\
\hline (Sub, Align) & 12 & 6 & 50 \\
\hline (Sub, Not Align) & 12 & $6 / 9$ & $50 / 25$ \\
\hline (Sub, Sub) & 27 & 15 & 44.4 \\
\hline Average & 13.2 & $6.8 \sim 6.9$ & $49.1 \sim 48.3$ \\
\hline
\end{tabular}

TABLE III

PSNR COMPARISON

\begin{tabular}{|c|c|c|c|c|}
\hline Sequence & Format & H.264 (dB) & Proposed (dB) & PSNR loss \\
\hline Akiyo & CIF & 43.72 & 41.16 & 2.56 \\
\hline Foreman & CIF & 41.23 & 39.20 & 2.03 \\
\hline Mobile & CIF & 37.61 & 34.14 & 3.47 \\
\hline Stefan & CIF & 38.82 & 34.88 & 3.94 \\
\hline Station & HDTV & 39.12 & 37.84 & 1.27 \\
\hline
\end{tabular}

The specifications of our H.264 decoder platform are $\mathrm{HD} 1080+\mathrm{HD} 720$ at $30 \mathrm{fps}$ with a working frequency at $150 \mathrm{MHz}$. The compressor converts a $4 \times 2$ block from the deblocking filter into a 32-bit segment, which is stored into the external memory device. Compared with the data access times of the external memory device for the system without EC, the data access times of our system are reduced by half. The decompressor converts a 32-bit segment into a $4 \times 2$ block, which is sent to MC. Since our system bus is 32 -bit wide and the external memory device is 32 bit per entry, the system accesses one data as four pixels.

In MC, accessing pixel data is based on the motion vector (MV). Moreover, the $x$ and $y$ values of an MV $(x, y)$ can be classified as follows: 1) Align if the value is quadruple and the required 4 pixels fit into the block grid; 2) Not Align if the value is not quadruple but is an integer and the required 4 pixels traverse two block grids; and 3) Sub if the value accuracy is either $1 / 2$ or $1 / 4$ precision. The required 9 pixels can be obtained by interpolating 4 neighboring pixels.

In Table II, we analyze the read access times of MC with/without EC. The worst case is the (Sub, Sub) case. To finish MC, a $4 \times 4$ block needs a $9 \times 9$ block. Therefore, the system with/without the proposed embedded compressor takes $15 / 27$ cycles. The best case is the (Align, Align) case. The original system with/without the embedded compressor needs $2 / 4$ cycles to finish the best case. For the other cases, when the required pixels of $\mathrm{MC}$ are not fit into $4 \times 2$ block grids, the access times become increased.

\section{EXPERIMENTAL RESULTS}

Table III shows the software simulation results of the proposed algorithm, which is integrated with JM16.2. Each test sequence executes 100 frames, such as Akiyo, Foreman, Mobile, Stefan, and Station, at ten frames for the I frame interval;
TABLE IV

COMPARISON WITH OTHER PREVIOUS WORK

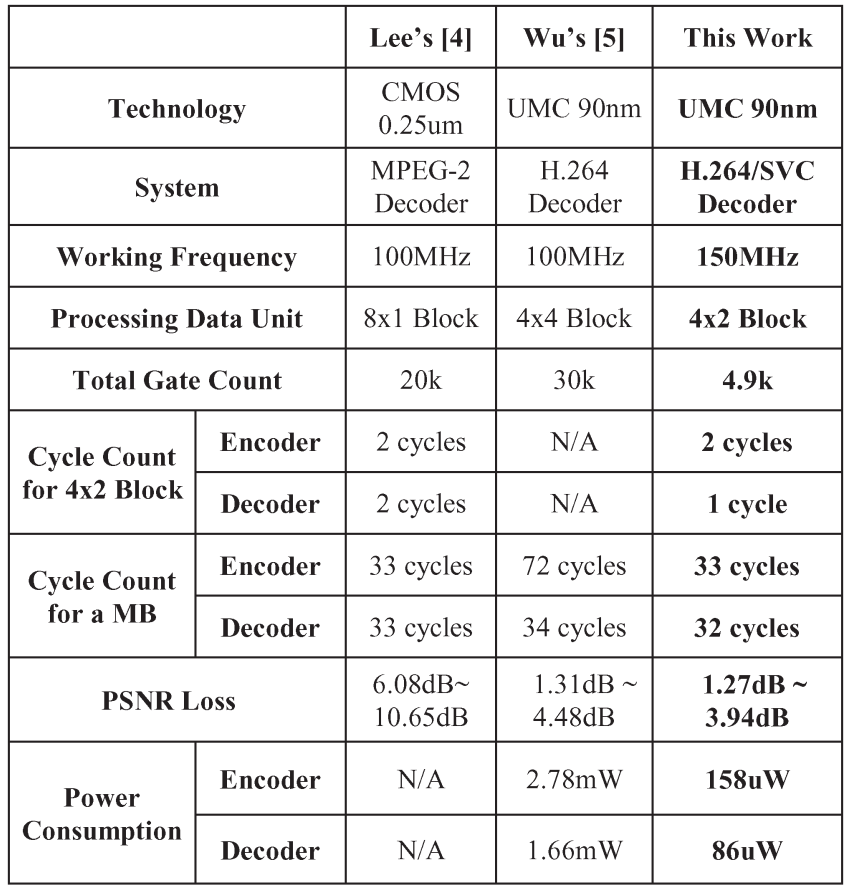

*The N/A is because of the processing data unit is $4 \times 4$ block in MBPZC

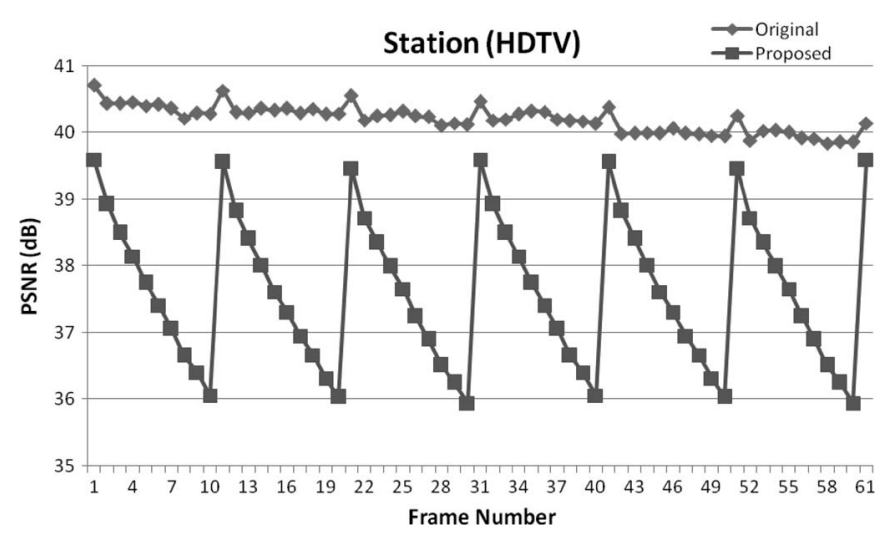

Fig. 10. Simulation result of the station sequence (in HDTV format).

quantization parameter is equal to 28; and the bit rate is set as $128 \mathrm{~kb} / \mathrm{s}$. The result shows that the peak-signal-to-noise-ratio (PSNR) loss is 1.27 to $3.94 \mathrm{~dB}$, compared with that from H.264. The proposed architecture is synthesized with 90-nm complementary metal-oxide-semiconductor standard-cell library, and the gate counts for the compressor and the decompressor are $4 \mathrm{k}$ and $0.9 \mathrm{k}$, respectively. The working frequency is up to $150 \mathrm{MHz}$ at HD1080/720. For power consumption, the compressor is $158 \mu \mathrm{W}$, and the decompressor is $86 \mu \mathrm{W}$.

Table IV shows the comparison with those from previous work. It can be found that our proposed hardware provides less hardware complexity and acceptable visual quality. In particular, the proposed decoder just requires one cycle with better random accessibility for EC without degrading the overall system performance. The power consumption of the proposed hardware is better than those in [4] and [5]. Fig. 10 shows the Station sequence result of the original system with EC in high definition television (HDTV) format. The propagation of 
visual quality loss is unavoidable, but the overall video quality remains acceptable.

\section{CONCLUSION}

In this brief, we have proposed a new EC algorithm for mobile video applications. With these advantages of the proposed EC algorithm, we can reduce the size of external memory and bandwidth utilization to achieve power saving. The pipelined architecture of the proposed decompressor requires 1 cycle; thus, the random accessibility becomes better. Due to the fixed $\mathrm{CR}$, the proposed $\mathrm{EC}$ algorithm is easier to be integrated with available video decoders such as the H.264 decoder.

\section{ACKNOWLEDGMENT}

The authors would like to thank the National Science Council of Taiwan for sponsoring this research and the members of the SI2 laboratory for their fruitful advice and assistance.

\section{REFERENCES}

[1] JVT G050, Draft ITU-T recommendation and final draft international standard of Joint Video Specification (ITU-T Rec. H264-ISO/IEC 1449610:2005 AVC), 2005.

[2] J. Kim and C. M. Kyung, "A lossless embedded compression using significant bit truncation for HD video coding," IEEE Trans. Circuits Syst. Video Technol., vol. 20, no. 6, pp. 848-860, Jun. 2010.

[3] X. Bao, D. Zhou, and S. Goto, "A lossless frame recompression scheme for reducing DRAM power in video encoding," in Proc. IEEE ISCAS, Jun. 2010, pp. 677-680.

[4] T. Y. Lee, "A new frame-recompression algorithm and its hardware design for MPEG-2 video decoders," IEEE Trans. Circuits Syst. Video Technol., vol. 13, no. 6, pp. 529-534, Jun. 2003.

[5] Y. D. Wu, Y. Li, and C. Y. Lee, "A novel embedded bandwidth-aware frame compressor for mobile video applications," in Proc. IEEE ISPACS, Feb. 2009, pp. 1-4.

[6] C. K. Yang and W. H. Tsai, "Improving block truncation coding by line and edge information and adaptive bit plane selection for gray-scale image compression," Pattern Recognit. Lett., vol. 16, no. 1, pp. 67-75, Jan. 1995.

[7] T. M. Amarunnishad, V. K. Govindan, and T. M. Abraham, "Block truncation coding using a set of predefined bit planes," in Proc. IEEE ICCIMA, Dec. 2007, vol. 3, pp. 73-78.

[8] T. M. Liu, C. C. Chung, C. Y. Lee, T. A. Lin, and S. Z. Wang, "A 125/spl mu/w, fully scalable MPEG-2 and H.264/AVC video decoder for mobile applications," in Proc. IEEE ISSCC, 2006, pp. 1576-1585. 